\title{
Thermal post-buckling of slender composite and FGM columns through a simple and novel FE formulation
}

\author{
G VENKATESWARA RAO ${ }^{1}$, K SANJAY ANANDRAO ${ }^{2, *}$ and R K GUPTA ${ }^{2}$ \\ ${ }^{1}$ Department of Mechanical Engineering, Sreenidhi Institute of Science and Technology, \\ Ghatkesar, Hyderabad 501 301, India \\ ${ }^{2}$ Advanced Systems Laboratory, Kanchanbagh, Hyderabad 500 058, India \\ e-mail: sanjayanandkhalane@gmail.com
}

MS received 8 June 2015; revised 22 November 2015; accepted 16 February 2016

\begin{abstract}
A simple and novel finite element (FE) formulation is proposed to study the thermal post-buckling of composite and FGM columns with axially immovable ends and operating in severe thermal environment. A linear eigenvalue analysis gives the critical buckling temperature but practically the buckled columns can withstand additional thermal load beyond critical temperature, which can be obtained using von-Karman geometric nonlinearity, applicable for moderately large deflections. In the present study, the solution of the nonlinear post-buckling problem is obtained by treating it as a linear eigenvalue problem using the concept of effective stiffness. Here, the total degrees of freedom (dof) of the discretized column are reduced and the postbuckling load is obtained without the need for iterative analysis. Comparison of the numerical results obtained from this FE formulation is in very good agreement with those obtained from the earlier FE formulations.
\end{abstract}

Keywords. Composite column; FGM column; finite element formulation; thermal post-buckling; vonKarman; geometric nonlinearity.

\section{Introduction}

Composites with its higher specific strength and stiffness are replacing metals in weight sensitive aerospace and automobile applications. Tailoring of the lay-up sequence of the composites, to suit the design requirements, is an additional advantage. In functionally graded material (FGM), two distinct materials are combined to form a structure with smooth and continuous change of microstructural composition and properties, generally along the thickness of beam/column or plate. Structural members such as columns/beams are basic components in many light weight applications of the structural systems. The columns/ beams, having axially immovable ends, experience axial compressive mechanical load, due to the high temperature rise from the initial stress free temperature when subjected to severe thermal environment. This compressive load causes instability of the column and causes large transverse deflection, before the column yields. A linear buckling analysis gives critical value of the induced mechanical load (also called thermal load) of the column, with the prescribed boundary conditions, on the transverse deflection or its derivative, at its ends. However, the column will be able withstand considerable additional compressive load after the initial instability (thermal buckling), before the ultimate

*For correspondence load is reached. The behavior of the column after buckling is studied by obtaining its thermal post-buckling load, so as to effectively utilize the additional compressive load carrying capability. The post-buckling behavior is obtained, by using the von-Karman nonlinear strain-displacement relation, if the transverse deflections of the column are of the order of the column thickness. Further, a proper care has to be taken, in the stiffness based design of the column that the high transverse deflections should not interfere with the functional requirements of the structural systems.

Buckling aspects of isotropic and homogenous columns, plates and shells are exhaustively reported by Timoshenko and Gere [1]. Dym [2] reported stability theory and its applications to structural mechanics. Thermal post-buckling analysis of slender, homogenous and isotropic columns using Galerkin finite element formulation is reported by Rao and Raju [3]. Rao and Raju [4] also studied the thermal post-buckling phenomenon of homogenous columns using the Rayleigh-Ritz method. In these studies, the thermal buckling load and the corresponding nonlinear thermal load parameters have been reported for the hinged, clamped and hinged-clamped columns. Rao and Raju [5, 6] presented a simple intuitive method to study the thermal post-buckling behavior of uniform columns. Gupta et al [7] carried out a comparative study of thermal post-buckling analysis of columns using an equilibrium path method and an iterative equivalent eigenvalue problem. Gupta et al [8] studied the 
thermal post-buckling analysis of composite columns to obtain simple and accurate closed form expressions. Anandrao et al $[9,10]$ studied thermal post-buckling of slender as well as shear flexible FGM columns using the classical Rayleigh-Ritz and the finite element method. Raju and Rao [11] studied thermal post-buckling of tapered columns using finite element method. Rao [12] and Raju and Rao [13] also studied thermal post-buckling of columns and plates resting on elastic foundation.

As mentioned previously, the thermal post-buckling analysis of slender columns has been reported earlier using the analytical and the numerical methods. The iterative formulation [4] requires four dof at each node $\left(u, u^{\prime}, w\right.$ and $\left.w^{\prime}\right)$ and the element non-linear stiffness matrix is of the order $8 \times 8$. The non-linear formulation [9] requires three dof at each node $\left(u, w\right.$ and $\left.w^{\prime}\right)$ and the element non-linear stiffness matrix is of the order $6 \times 6$. In the present work, a novel formulation based on the FE method is proposed to study the thermal post-buckling of slender composite and FGM columns subjected to uniform temperature increment along length and across thickness, from the initial stress free temperature. In this formulation, the numbers of degrees of freedom at each node are reduced to two ( $w$ and $\left.w^{\prime}\right)$ since the nodal dof corresponding to the axial displacement need not be considered in the analysis. The element stiffness matrix is of the order $4 \times 4$ thus reducing the order of the assembled matrices. Further, in the conventional nonlinear analysis [9], the stiffness matrix is updated after each iteration based on the earlier deflection state, in the iterative solution procedure. However, in the present novel FE formulation, the iterative procedure is not required, because of the use of the concept of the effective stiffness matrix, which reduces the computational time significantly. In this study, a column with classical boundary conditions is considered and the post- buckling results in terms of non-linear to linear thermal post-buckling loads for various normalized transverse deflection are compared with the existing literature results to bring out the accuracy of the present formulation with reduced computational efforts.

\section{Material properties and lay-ups}

A Carbon/Epoxy unidirectional composite lamina with the material properties as given in table 1 is considered in the present study. Four different lay-up sequences and the corresponding stiffnesses considered in the analysis are given in table 2. The total thickness ' $h$ ' of laminates for each lay-up is considered as $1 \mathrm{~mm}$.

A typical composite column with lay-up 3 is shown in figure 1.

A FGM column as shown in figure 2 with ceramic (Zirconia) on top face and metal (Aluminium) on bottom face with material properties as given in table 3 is considered in this study. The variation of properties $E$ and $\alpha$ is governed by a power law distribution, as given in Eq. (1), with the co-ordinate $z$ along thickness of column varying between $-h / 2$ and $h / 2$. The volume fraction exponent $n$ can take any value between 0 and $\infty$, with $n=0$ and $n=\infty$ corresponding to the two extremes of completely homogenous ceramic and metal beams respectively.

$$
\begin{gathered}
E(z)=E_{c} V_{c}+E_{m}\left(1-V_{c}\right) \\
V_{c}=(0.5+z / h)^{n} .
\end{gathered}
$$

Figure 3 shows variation of Young's modulus for various volume fraction exponents ' $n$ '.

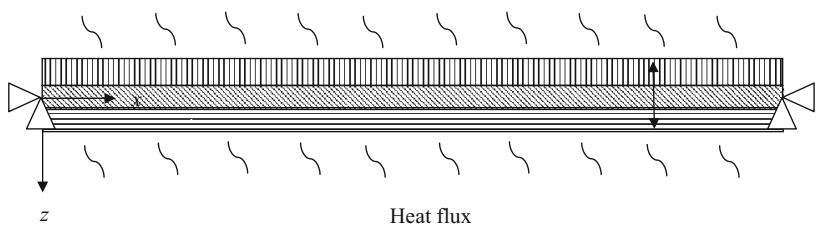

Figure 1. Composite column with lay-up 3.
Table 1. Material properties for Carbon/Epoxy composite.

\begin{tabular}{lc}
\hline Property & Value \\
\hline$E_{11}$ & $100 \mathrm{GPa}$ \\
$E_{22}$ & $6.2 \mathrm{GPa}$ \\
$v_{12}$ & 0.29 \\
$G_{12}$ & $4.0 \mathrm{GPa}$ \\
\hline
\end{tabular}

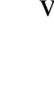




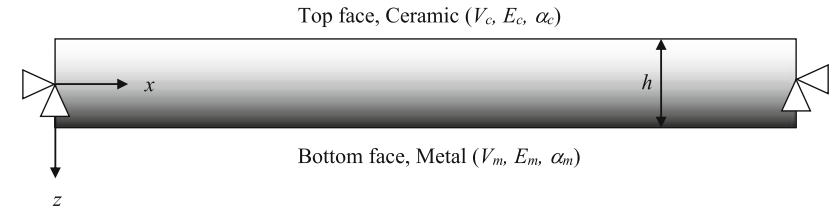

Figure 2. FGM column with ceramic and metal as constituents.

Table 3. Material properties of FGM (Zirconia and Aluminium).

\begin{tabular}{lcc}
\hline Property & Zirconia & Aluminium \\
\hline$E(\mathrm{GPa})$ & 151 & 70 \\
$\alpha(/ \mathrm{K})$ & $10 \mathrm{e}-06$ & $23 \mathrm{e}-06$ \\
\hline
\end{tabular}

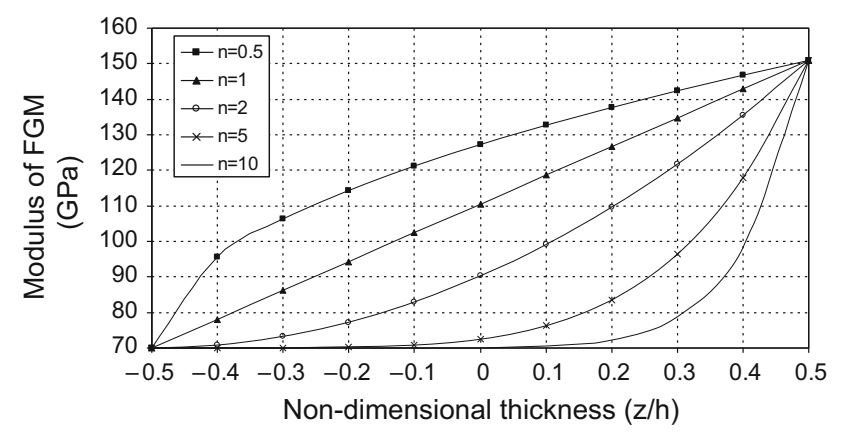

Figure 3. Variation of Young's Modulus across thickness of FGM beam.

\section{Finite element formulation}

A finite element formulation for laminated composite beams based on Euler-Bernoulli beam theory is developed [14]. A slender beam/column is considered so that the effect of transverse shear on the deformation of the column can be neglected. A two node beam element with two degrees of freedom per node (transverse deflection $w$ and slope $w^{\prime}=\frac{\mathrm{d} w}{\mathrm{~d} x}$ ) is considered. The dof vector for the beam is written as

$$
w(x)=H_{1} w_{1}+H_{2} \frac{\mathrm{d} w_{1}}{\mathrm{~d} x}+H_{3} w_{2}+H_{4} \frac{\mathrm{d} w_{2}}{\mathrm{~d} x} .
$$

In the present formulation, the axial degree of freedom ' $u_{i}$ ' need not be considered as explained later in this section. The differential equation governing the buckling of straight beam is

$$
\frac{\mathrm{d}}{\mathrm{d} x}\left(\frac{\mathrm{d} M_{x x}}{\mathrm{~d} x}+N_{x x} \frac{\mathrm{d} w_{0}}{\mathrm{~d} x}\right)=0
$$

The stress and moment resultants in the axial direction can be expressed as

$$
\begin{aligned}
& N_{x x}=A_{11} \varepsilon_{x}^{0}+B_{11} \kappa_{x} \\
& M_{x x}=B_{11} \varepsilon_{x}^{0}+D_{11} \kappa_{x}
\end{aligned}
$$

where ' $A_{11}$ ', ' $B_{11}$ ' and ' $D_{11}$ ' for composite column are given by

$$
\begin{aligned}
& A_{11}=\sum_{k=1}^{N}\left(\bar{Q}_{11}\right)_{k}\left(z_{k}-z_{k-1}\right) \\
& B_{11}=\frac{1}{2} \sum_{k=1}^{N}\left(\bar{Q}_{11}\right)_{k}\left(z_{k}^{2}-z_{k-1}^{2}\right) \\
& D_{11}=\frac{1}{3} \sum_{k=1}^{N}\left(\bar{Q}_{11}\right)_{k}\left(z_{k}^{3}-z_{k-1}^{3}\right) .
\end{aligned}
$$

$\bar{Q}_{11}$ is transformed reduced stiffness given in terms of $Q_{11}$ as

$$
\bar{Q}_{11}=Q_{11} \cos ^{4} \theta+2\left(Q_{12}+2 Q_{66}\right) \sin ^{2} \theta \cos ^{2} \theta+Q_{22} \sin ^{4} \theta
$$

and

$$
\begin{gathered}
Q_{11}=\frac{E_{11}}{1-v_{12} v_{21}} \\
Q_{12}=\frac{v_{12} E_{2}}{1-v_{12} v_{21}} \\
Q_{22}=\frac{E_{2}}{1-v_{12} v_{21}} \\
Q_{66}=G_{12} \\
\frac{v_{12}}{E_{11}}=\frac{v_{21}}{E_{22}} .
\end{gathered}
$$

For FGM column, ' $A_{11}$ ', ' $B_{11}$ ' and ' $D_{11}$ ' are given by

$$
\begin{aligned}
A_{11} & =\int_{-h / 2}^{h / 2} E(z) \mathrm{d} z \\
B_{11} & =\int_{-h / 2}^{h / 2} E(z) z \mathrm{~d} z \\
D_{11} & =\int_{-h / 2}^{h / 2} E(z) z^{2} \mathrm{~d} z .
\end{aligned}
$$

By using the principle of virtual work, the weak form of the governing equation of motion can be obtained. Substituting the expressions for the dof vector in the weak form, the FE system of equations can be obtained [14].

In the case of the buckling of the composite column, with axially immovable ends, subjected to a uniform 
temperature rise, above the ambient stress free temperature through the thickness, the following eigenvalue problem is obtained as

$$
[K]\left\{\delta_{L}\right\}-\lambda_{L}[G]\left\{\delta_{L}\right\}=0 .
$$

By solving the above finite element system of equations, mechanical equivalent of the thermal load ' $P_{L}$ ', which will cause instability in the column is obtained.

The next step in the present FE formulation is to obtain an expression for tensile load. The tensile load ' $T$ ' induced in the column with axially immovable ends due to moderately large transverse deflections is evaluated by equating the outward and inward axial displacements as explained.

For a tensile load ' $T$ ' applied on one end of column (where axial immovability condition is removed), the outward axial displacement ' $u_{0}$ ' is

$$
u_{0}=\frac{T L}{A E_{11}}
$$

and

$$
E_{11}=\frac{A_{11}}{h} .
$$

The strain-displacement relations considering von-Karman type geometric nonlinearity are given by

$$
\begin{gathered}
\varepsilon_{x x}^{0}=\frac{\mathrm{d} u_{0}}{\mathrm{~d} x}+\frac{1}{2}\left(w^{\prime}\right)^{2} \\
\kappa_{x x}=-\frac{\mathrm{d}^{2} w_{0}}{\mathrm{~d} x^{2}} \\
\varepsilon_{x x}=\varepsilon_{x x}^{0}+z \kappa_{x x} .
\end{gathered}
$$

If the column is undergoing moderately large transverse deflections, then the inward axial displacement ' $u_{i}$ ' at this end (where the axially immovability condition is removed) is obtained by equating the first equation above, which represents axial strain at Neutral Axis, to zero and integrating as

$$
\begin{gathered}
\varepsilon_{x x}^{0}=\frac{\mathrm{d} u_{0}}{\mathrm{~d} x}+\frac{1}{2}\left(w^{\prime}\right)^{2}=0 \\
u_{i}=\frac{1}{2} \int_{0}^{L}\left(w^{\prime}\right)^{2} \mathrm{~d} x .
\end{gathered}
$$

Equating the magnitudes of the outward and inward axial displacement of the column from Eq. (10a) and Eq. (12), the tensile load is obtained as

$$
T=\frac{A E_{11}}{2 L} \int_{0}^{L}\left(w^{\prime}\right)^{2} \mathrm{~d} x .
$$

The above equation represents that the two ends of the column are axially immovable and Eqs (10a) and (12) are used for evaluating the axial tension ' $T$ ' developed in the column due to moderately large transverse deflection in terms of the slope of transverse deflection $w$. The necessity of considering the axial displacement dof ' $u$ ' is thus eliminated in evaluating tensile load ' $T$ ' in this formulation. The material parameter $\left(E_{11}\right)$ and geometric parameters $(A$ and $L$ ) of the column and the information of the slope, at each node are sufficient to evaluate ' $T$ '. For a column with axially immovable ends, this tensile load will cause change in the stiffness of the column and can be utilized to obtain the effective stiffness and then the post-buckling load. The thermal post-buckling problem of the composite columns can now be expressed in a matrix form, as

$$
\begin{gathered}
{\left[K_{E}\right]=[K]+T[G]} \\
{\left[K_{E}\right]\left\{\delta_{N L}\right\}-\lambda_{N L}[G]\left\{\delta_{N L}\right\}=0}
\end{gathered}
$$

where $\lambda_{N L}$ is the nonlinear eigenvalue (thermal post-buckling load) and $\left\{\delta_{N L}\right\}$ is the nonlinear eigenvector. The nonlinear eigenvector is scaled so that the central transverse deflection will have a value of ' $a$ ' which is normalized with the radius of gyration ' $r$ ' to obtain the ratio of $\frac{a}{r}$. It is to be noted here that no iterative procedure is required to be followed to obtain the thermal post-buckling load.

\section{Results and discussion}

A convergence study is carried out for a clamped-clamped and hinged-hinged column with different number of elements $N E$ to obtain the ratio of the thermal post-buckling to the thermal buckling load $\frac{P_{N L}}{P_{L}}$. A slender column with height $h=1 \mathrm{~mm}$ and $\frac{L}{h}=60$ is used in this study. The width of column is assumed to be unity. The Simpson's rule is used to numerically integrate and evaluate the axial displacement from the nodal values of slope of transverse deflection $\left(\frac{\mathrm{d} w}{\mathrm{~d} x}\right)$ of the column obtained from the eigenvector $\left\{\delta_{N L}\right\}$. This non-linear eigenvector is obtained by multiplying the eigenvector obtained from Eq. (9) with the central transverse deflection ' $a$ '.

$$
u_{i}=\frac{1}{2} \int_{0}^{L}\left(w^{\prime}\right)^{2} \mathrm{~d} x
$$

The tensile load ' $T$ ' is evaluated using Eq. (13) and substituted in Eq. (14) to get the effective stiffness matrix $\left[K_{E}\right]$. Eq. (14) can then be solved as an eigenvalue problem to obtain thermal post-buckling load ' $P_{N L}$ ' and the corresponding nonlinear eigenvector $\left\{\delta_{N L}\right\}$. The linear thermal buckling load can be evaluated by using Eq. (9) or Eq. (14) with $\frac{a}{r}=0$. Tables 4 and 5 show the results of convergence study for lay-up 2 for $\frac{a}{r}=1$. 
Table 4. Convergence study for Clamped-Clamped composite column.

\begin{tabular}{lcclll}
\hline$N E$ & $P_{L}$ & $\lambda_{L}=\frac{P_{L} L^{2}}{E I}$ & \multicolumn{1}{c}{$T$} & $\lambda_{T}=\frac{T L^{2}}{E I}$ & \multicolumn{1}{c}{$\frac{P_{N L}}{P_{L}}$} \\
\hline 2 & 93.0778 & 40.0096 & 0 & 0 & 1.0 \\
4 & 92.5551 & 39.7849 & 7.6240 & 3.2772 & 1.0824 \\
8 & 91.9111 & 39.5081 & 5.7398 & 2.4673 & 1.0624 \\
16 & 91.8671 & 39.4891 & 5.7401 & 2.4674 & 1.0625 \\
\hline
\end{tabular}

Table 5. Convergence study for Hinged-Hinged composite column.

\begin{tabular}{lccccc}
\hline$N E$ & $P_{L}$ & $\lambda_{L}=\frac{P_{L} L^{2}}{E I}$ & $T$ & $\lambda_{T}=\frac{T L^{2}}{E I}$ & $\frac{P_{N L}}{P_{L}}$ \\
\hline 2 & 23.1388 & 9.9462 & 3.8120 & 1.6386 & 1.1647 \\
4 & 22.9778 & 9.8770 & 5.7398 & 2.4673 & 1.2498 \\
8 & 22.9668 & 9.8723 & 5.7401 & 2.4674 & 1.2499 \\
16 & 22.9661 & 9.8720 & 5.7401 & 2.4674 & 1.2499 \\
\hline
\end{tabular}

The column is discretised with equal length elements. The results with 16 elements idealization of the composite column is observed to give converged ratio $\frac{P_{N L}}{P_{L}}$ and is used for the further analysis. The convergence of the ratio $\frac{P_{N L}}{P_{L}}$ with increasing number of elements is found to be very good.

The validation of the present novel formulation is carried out considering the homogenous, isotropic and composite columns. Tables 6 and 7 show the comparison of the ratio of the thermal post-buckling to thermal buckling load $\frac{P_{N L}}{P_{L}}$ for various $\frac{a}{r}$ and for various boundary conditions. In the absence of any geometric non-linearity, the transverse deflection of the column will be zero and the non-linear thermal load will be same as linear thermal load. An excellent match is found for the ratio of thermal postbuckling to the buckling load for all the ratios of $\frac{a}{r}$ considered in the present analysis.

The study is extended to obtain the thermal post-buckling load for all the composite lay-ups. Tables 8 and 9 show the ratio of non-linear to linear thermal buckling load $\frac{P_{N L}}{P_{L}}$ for various lay-ups at different $\frac{a}{r}$ and for various boundary conditions.

The study is extended for FGM column with clamped ends. Figure 4 shows the pre-buckling and post-buckling load deformation paths with various volume fraction exponents. A very good agreement can be observed

Table 6. Post-buckling ratio for Clamped-Clamped column.

\begin{tabular}{|c|c|c|c|c|c|c|}
\hline \multirow[b]{3}{*}{$a / r$} & \multicolumn{6}{|c|}{$P_{N L} / P_{L}$} \\
\hline & \multicolumn{2}{|c|}{ Isotropic } & \multicolumn{2}{|c|}{ Layup 2} & \multicolumn{2}{|c|}{ Layup 4} \\
\hline & Gupta et al (2009) & Present & Gupta et al (2010) & Present & Gupta et al (2010) & Present \\
\hline 0.0 & 1.0000 & 1.0000 & 1.0000 & 1.0000 & 1.0000 & 1.0000 \\
\hline 0.2 & 1.0025 & 1.0025 & 1.0025 & 1.0025 & 1.0018 & 1.0018 \\
\hline 0.4 & 1.0100 & 1.0100 & 1.0100 & 1.0100 & 1.0071 & 1.0071 \\
\hline 0.6 & 1.0225 & 1.0225 & 1.0225 & 1.0225 & 1.0160 & 1.0160 \\
\hline 0.8 & 1.0400 & 1.0400 & 1.0400 & 1.0400 & 1.0285 & 1.0285 \\
\hline 1.0 & 1.0625 & 1.0625 & 1.0625 & 1.0625 & 1.0445 & 1.0445 \\
\hline 2.0 & 1.2500 & 1.2500 & 1.2500 & 1.2499 & 1.1781 & 1.1780 \\
\hline 3.0 & 1.5625 & 1.5625 & 1.5625 & 1.5623 & 1.4006 & 1.4006 \\
\hline
\end{tabular}

Table 7. Post-buckling ratio for Hinged-Hinged column.

\begin{tabular}{|c|c|c|c|c|c|c|}
\hline \multirow[b]{3}{*}{$a / r$} & \multicolumn{6}{|c|}{$P_{N L} / P_{L}$} \\
\hline & \multicolumn{2}{|c|}{ Isotropic } & \multicolumn{2}{|c|}{ Layup 2} & \multicolumn{2}{|c|}{ Layup 4} \\
\hline & Gupta et al (2009) & Novel FEM & Gupta et al (2010) & Novel FEM & Gupta et al (2010) & Novel FEM \\
\hline 0.0 & 1.00 & 1.00 & 1.0000 & 1.0000 & 1.0000 & 1.0000 \\
\hline 0.2 & 1.01 & 1.01 & 1.0100 & 1.0100 & 1.0071 & 1.0071 \\
\hline 0.4 & 1.04 & 1.04 & 1.0400 & 1.0400 & 1.0285 & 1.0285 \\
\hline 0.6 & 1.09 & 1.09 & 1.0900 & 1.0900 & 1.0641 & 1.0641 \\
\hline 0.8 & 1.16 & 1.16 & 1.1600 & 1.1600 & 1.1140 & 1.1140 \\
\hline 1.0 & 1.25 & 1.25 & 1.2499 & 1.2499 & 1.1781 & 1.1781 \\
\hline 2.0 & 2.00 & 2.00 & 1.9998 & 1.9998 & 1.7122 & 1.7122 \\
\hline 3.0 & 3.25 & 3.25 & 3.2495 & 3.2495 & 2.6025 & 2.6025 \\
\hline
\end{tabular}


Table 8. Post-buckling ratio for Clamped-Clamped composite column.

\begin{tabular}{lcccc}
\hline & \multicolumn{4}{c}{$P_{N L} / P_{L}$} \\
\cline { 2 - 5 }$a / r$ & Layup 1 & Layup 2 & Layup 3 & Layup 4 \\
\hline 0.0 & 1.0000 & 1.0000 & 1.0000 & 1.0000 \\
0.2 & 1.0061 & 1.0025 & 1.0048 & 1.0018 \\
0.4 & 1.0243 & 1.0100 & 1.0193 & 1.0071 \\
0.6 & 1.0547 & 1.0225 & 1.0435 & 1.0160 \\
0.8 & 1.0972 & 1.0400 & 1.0773 & 1.0285 \\
1.0 & 1.1519 & 1.0625 & 1.1208 & 1.0445 \\
2.0 & 1.6077 & 1.2499 & 1.4831 & 1.1780 \\
3.0 & 2.3674 & 1.5623 & 2.0870 & 1.4006 \\
\hline
\end{tabular}

Table 9. Post-buckling ratio for Hinged-Hinged composite column.

\begin{tabular}{lcccc}
\hline & \multicolumn{4}{c}{$P_{N L} / P_{L}$} \\
\cline { 2 - 5 }$a / r$ & Layup 1 & Layup 2 & Layup 3 & Layup 4 \\
\hline 0.0 & 1.0000 & 1.0000 & 1.0000 & 1.0000 \\
0.2 & 1.0122 & 1.0100 & 1.0104 & 1.0071 \\
0.4 & 1.0490 & 1.0400 & 1.0416 & 1.0285 \\
0.6 & 1.1102 & 1.0900 & 1.0936 & 1.0641 \\
0.8 & 1.1959 & 1.1600 & 1.1663 & 1.1140 \\
1.0 & 1.3062 & 1.2499 & 1.2599 & 1.1781 \\
2.0 & 2.2247 & 1.9998 & 2.0394 & 1.7122 \\
3.0 & 3.7555 & 3.2495 & 3.3388 & 2.6025 \\
\hline
\end{tabular}

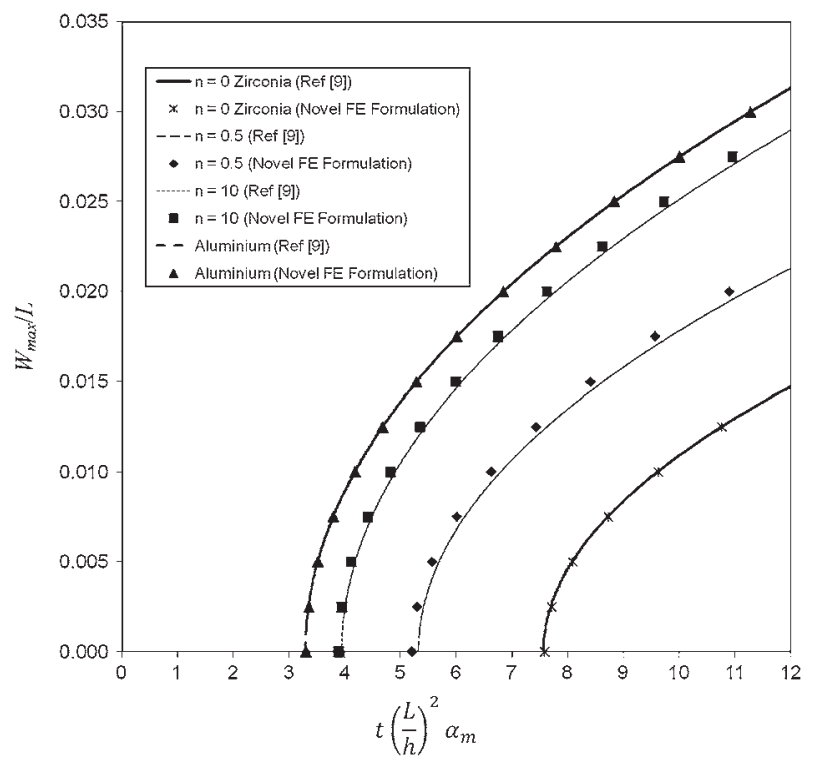

Figure 4. Load deformation curve for Clamped-Clamped FGM Beam. between the results obtained with novel formulation and literature results.

\section{Conclusions}

In the present study, a very simple and elegant novel finite element formulation is used to predict the thermal postbuckling load of composite and FGM columns for various classical boundary conditions. The main aim of the present study is to use standard linear element stiffness matrix and geometric stiffness matrix developed using nodal transverse deflection and its slope, to obtain the thermal post-buckling load. This is achieved without the need for considering nodal axial degree of freedom and its derivative as required for earlier formulations. This reduces the order of matrices required to solve the problem. Further, the need for iterative analysis is also eliminated thus reducing the computational time. It is demonstrated in this study that the above benefits in terms of reduced order of matrices and computational time are obtained with the same accuracy of results of the earlier developed formulations.

\section{Nomenclature}

$a$

A

$A_{11}$

$B_{11}$

$D_{11}$

$E_{11}$

$E_{22}$

E

$v_{12}, v_{21}$

$G_{12}$

[G]

$h$

$H_{i}$

I

$[K]$

$\left[K_{E}\right]$

$L$

$M_{x x}$

$N$

$N_{x x}$

$N E$

P

$Q_{11}$

$\bar{Q}_{11}$

$n$

$r$ central transverse deflection

cross sectional area

extensional stiffness

bending-extension coupling stiffness

bending stiffness

elastic modulus in fiber direction

elastic modulus in transverse direction

effective modulus of the column $\left(=\frac{A_{11}}{t}\right)$

in-plane major and minor Poisson ratio

in-plane shear modulus

geometric stiffness matrix

thickness of column

Hermite shape functions

area moment of inertia of the column crosssection

linear elastic stiffness matrix

effective stiffness matrix

length of column

moment resultant

total number of layers

stress resultant

number of elements

mechanical equivalent of the thermal load

reduced stiffness

transformed reduced stiffness

volume fraction exponent

radius of gyration of the column cross-section 
temperature increment

tensile load induced in column due to large transverse deflections

$u$

$u^{\prime}$

V

w

$w^{\prime}$

$x$

$z$

$z_{k}, z_{k-1}$

$\varepsilon_{x x}$

$\varepsilon_{x x}^{0}$

$x x$

$\theta$

$\lambda$

$\lambda_{L}, \lambda_{T}$

$\{\delta\}$

$\alpha$

axial displacement

$\mathrm{d} u / \mathrm{d} x$

volume fraction

transverse deflection of column

$\mathrm{d} w / \mathrm{d} x$

coordinate along axis of column

coordinate along thickness of column

depth coordinates of a particular layer

axial strain

axial strain at Neutral Axis

curvature

angle made by a layer with respect to the axial direction $x$

eigenvalue

linear buckling load parameter, tension parameter

eigenvector (buckled mode shape)

coefficient of linear thermal expansion

\section{Subscripts}

$\begin{array}{ll}1,2 & \text { node numbers } \\ L & \text { linear } \\ N L & \text { nonlinear } \\ m, c & \text { metal, ceramic } \\ 0 & \text { mid-thickness } \\ 11,22 & \text { fiber and transverse directions }\end{array}$

\section{References}

[1] Timoshenko S P and Gere J M 1961 Theory of elastic stability. McGraw-Hill, New York

[2] Dym C L 1964 Stability theory and its application to structural mechanics. Noordhoff International, Leyden

[3] Rao G V and Raju P C 1977 Post-buckling of uniform cantilever columns - Galerkin finite element formulation. Eng. Fract. Mech. 9: 1-4

[4] Rao G V and Raju K K 1984 Thermal post buckling of columns. AIAA J 22(6): 850-851

[5] Rao G V and Raju K K 2002 Thermal post buckling of uniform Columns: A simple intuitive method. AIAA J., 40(10): 2138-2140

[6] Rao G V and Raju K K 2003 A simple method to predict the thermal post-buckling behavior of columns on Pasternak foundation. Indian J. Eng. Mater. Sci. 10: 177-182

[7] Gupta R K, Anandrao K S, Jagadish B, Janardhan G R and Rao G V 2009 Comparative study of thermal post-buckling analysis of columns using an equilibrium path method and an equivalent eigenvalue problem. IES J. A: Civil Struct. Eng. 2(4): 296-303

[8] Gupta R K, Jagadish B, Janardhan G R and Rao G V 2010 Postbuckling analysis of composite columns: Simple and accurate closed form expressions. Compos. Struct. 92: 1947-1956

[9] Anandrao K Sanjay, Gupta R K, Ramchandran P and Rao G V 2010 Thermal post-buckling analysis of uniform slender functionally graded material columns. Struct. Eng. Mech. Int. J. 36(5): 545-560

[10] Anandrao K Sanjay, Gupta R K, Ramchandran P and Rao G V 2012 Non-linear free vibrations and post-buckling analysis of shear flexible functionally graded columns. Struct. Eng. Mech. Int. J. 44(3): 1-23

[11] Raju K K and Rao G V 1984 Finite element analysis of thermal post-buckling of tapered columns. Comput. Struct. 19: $617-620$

[12] Rao G V 2003 A simple energy method to predict the thermal post buckling behavior of columns. J. Aerospace Sci. Technol. 55(2): 141-143

[13] Raju K K and Rao G V 1988 Thermal post-buckling of a square plate resting on an elastic foundation by finite element method. Comput. Struct. 28: 195-199

[14] Reddy J N 2003 Mechanics of laminated composite plates and shells: Theory and analysis. CRC Press, New York, 131-166 